\title{
Diagnóstico posnatal de hematoma de cordón umbilical. Reporte de un
} caso

\section{Postnatal diagnosis of umbilical cord hematoma. Case report}

\section{Cristina Rosales-Torbaño ${ }^{1}$, Elena Cobo-Nieto ${ }^{1}, Z_{\text {Z }}$ Zoraida Frías-Sánchez ${ }^{1 *}$ y Mónica Marín-Cid²}

${ }^{1}$ Unidad de Gestión Clínica de Ginecología y Patología Mamaria; ${ }^{2}$ Unidad de Gestión Clínica de Medicina Maternofetal. Hospital Universitario Virgen del Rocío, Sevilla, España

\section{Resumen}

Antecedentes: El hematoma espontáneo del cordón umbilical es una complicación rara del embarazo que representa una causa grave de morbilidad y mortalidad fetal. Se han descrito numerosos factores de riesgo, pero aún se desconoce su etiología exacta. Caso clínico: Mujer de 28 años, en su octava gestación, que consultó en urgencias por disminución de los movimientos fetales de varias horas de evolución tras una caída accidental traumática. La monitorización fetal mostró un patrón cardiotocográfico no tranquilizador, con disminución de la variabilidad y sin aceleraciones. Se realizó una cesárea urgente, sin complicaciones, con el nacimiento de un varón vivo de $4560 \mathrm{~g}$, con Apgar 8/10/10 y pH de sangre de cordón umbilical 7.08, que precisó ingreso en la unidad de neonatología por hallazgo de un soplo cardíaco. Tras el alumbramiento se objetivó una gran colección hemática en el cordón umbilical. Método: Se aplicó una estrategia de búsqueda sistemática en Medline, PubMed y Cochrane de todos los artículos en inglés y español que tuvieran como palabras clave "Umbilical", "Cordón" " "Hematoma". Resultados: Se encontraron 13 publicaciones de 15 casos de hematoma del cordón umbilical en los años 2008-2020. Se realizó una revisión sistemática de los informes de casos descritos en los últimos 12 años en la literatura para evaluar la epidemiología, los factores predisponentes, los resultados potenciales, el diagnóstico prenatal y el manejo clínico. Conclusiones: Debido a la rareza de esta afección, se deben informar todos los casos nuevos de hematoma del cordón umbilical para mejorar el conocimiento de los factores predisponentes, el diagnóstico prenatal y el manejo clínico.

Palabras clave: Cordón. Umbilical. Hematoma.

\section{Abstract}

Background: Spontaneous umbilical cord hematoma is a rare complication of pregnancy which represents a serious cause of fetal morbidity and mortality. There are many risk factors, but the exact etiology is still unknown. Case report: 28-year-old woman, eighth gestation, who consulted due to decreased fetal movements of several hours of evolution after traumatic accidental fall. Fetal monitoring showed a non-reassuring cardiotocographic pattern, with decreased fetal variability, without accelerations. An emergency cesarean section was performed without complications, with the birth of a living male weighing 4560 grams, with an 8/10/10 Apgar test, and an arterial pH of the umbilical cord 7.08, which required admission to the neonatology unit. After delivery, a large blood collection was observed in the umbilical cord. Method: A systematic search strategy was applied to several electronic bibliographic databases: Medline, PubMed and Cochrane. Key words used were "Umbilical","Cord"'“Hematoma". Results: 13 publications of 15 cases of umbilical cord hematoma were reported in the years 2008-2020. A systematic review of the reports of cases, described in the last twelve years in the literature was carried out to

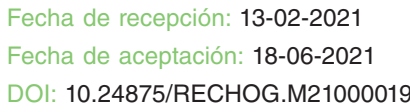

Rev Chil Obstet Ginecol. 2021;86(4):397-402

www. rechog.com 
evaluate the epidemiology, predisposing factors, potential results, prenatal diagnosis and clinical management of this phenomenon. Conclusions: Due to the rarity of this condition, every new case of umbilical cord hematoma should be reported in order to improve the knowledge of predisposing factors, prenatal diagnosis, and clinical management.

Key words: Cord. Umbilical. Hematoma.

\section{Introducción}

El hematoma espontáneo del cordón umbilical es un trastorno poco frecuente. La mayoría están localizados cerca del ombligo y suelen producirse por la rotura de la vena umbilical. Su etiología se desconoce, aunque se han descrito múltiples asociaciones, siendo las más frecuentes las infecciones amnióticas, los traumatismos, los cordones cortos, las formaciones aneurismáticas y los procedimientos invasivos, como la funiculocentesis ${ }^{1}$. Generalmente son pequeños, están bien circunscritos y suelen pasar inadvertidos. Los de gran tamaño se asocian con importantes secuelas neurológicas y con una tasa de mortalidad perinatal cercana al 50\%, que suele deberse a hipoxia fetal, secundaria a la interrupción del flujo sanguíneo en las arterias umbilicales, o a hipovolemia ${ }^{2}$. El diagnóstico suele ser un hallazgo posnatal inesperado. El diagnóstico prenatal depende del tamaño y de su orientación en el útero, y se requiere un alto índice de sospecha. La visualización de una masa intrauterina adyacente al abdomen fetal debe alertar sobre su presencia. La finalización de la gestación tras alcanzar la madurez fetal o el seguimiento ecográfico estrecho son las únicas medidas que pueden adoptarse para evitar las lesiones hipóxicas-isquémicas irreversibles o la muerte fetal intrauterina ${ }^{3}$.

A continuación, revisamos la literatura científica a propósito del caso de una gestante a término que consultó en urgencias por disminución de los movimientos fetales. La monitorización fetal mostró un patrón cardiotocográfico no tranquilizador, con disminución de la variabilidad y sin presentar aceleraciones.

\section{Método}

Se realizó una revisión de la literatura para identificar los informes de casos publicados. Se realizaron búsquedas en las bases de datos electrónicas. Se realizaron búsquedas en las bases de datos electrónicas PubMed, Medline y Cochrane de publicaciones entre 2008 y 2020 con las palabras clave "Umbilical", "Cordón" y "Hematoma" (se usaron diferentes combinaciones de los términos). Se eligió el año 2008 como punto de partida para la búsqueda porque en ese año
Gualandri, et al. ${ }^{4}$ hicieron una revisión de la literatura de los años 1958 a 2008. De las descripciones de los autores de informes de casos individuales tomamos la información disponible y confiable sobre los posibles factores predisponentes, la presentación clínica, el diagnóstico y el manejo (Tabla 1). Debido a la falta de uniformidad en los casos revisados, no se pudo realizar ningún cálculo con significación estadística.

\section{Caso clínico}

Mujer de 28 años que fue valorada en urgencias de obstetricia y ginecología del Hospital Universitario Virgen del Rocío, en Sevilla, España.

La paciente presentaba como antecedentes médicos de interés diabetes mellitus tipo 1 subtipo LADA (Latent Autoimmune Diabetes in Adults), con seguimiento errático y mala adherencia al tratamiento. Se trataba de su octava gestación, con historia de tres abortos espontáneos y cuatro partos vaginales. Los recién nacidos presentaron pesos fetales por encima de $4000 \mathrm{~g}$. En el último parto tuvo lugar una distocia de hombros que provocó una fractura de húmero en el feto. Esta nueva gestación no fue programada, y se inició con cifras de hemoglobina glucosilada en torno al 12\%. Se realizó una valoración periódica de la gestación en las consultas de alto riesgo obstétrico, permaneciendo con cifras de glucemia elevadas a pesar del tratamiento con insulina.

Además, desde la semana 28 de gestación presentó polihidramnios asociado a un peso fetal estimado por encima del percentil 100. A las 36 semanas de gestación, la paciente acudió al servicio de urgencias por disminución de la percepción de los movimientos fetales, de 6 horas de evolución, tras una caída accidental en su domicilio, en la que se produjo un traumatismo abdominal leve al precipitarse hacia delante. No presentó sangrado, hidrorrea ni otra sintomatología de alarma. Se realizó una valoración global en la consulta, con exploración física general y obstétrica.

Presentaba buen estado general y los genitales externos eran normales. En la especuloscopia, el cuello uterino no mostraba alteraciones macroscópicas, se encontraba cerrado y no se visualizaban sangrado ni 
Tabla 1. Reporte de casos descritos en la literatura en la última década (2009-2020)

\begin{tabular}{|c|c|c|c|c|c|c|c|}
\hline Autores & Edad materna & $\begin{array}{l}\text { Edad } \\
\text { gestacional }\end{array}$ & Curso prenatal & Tipo de parto & Examen histológico & Resultado fetal & $\widetilde{\sim}$ \\
\hline \multirow[t]{3}{*}{$\begin{array}{l}\text { Towers, et al. } \\
\text { (2009) }\end{array}$} & 23 & 31 & $\begin{array}{l}\text { Disminución de } \\
\text { movimientos } \\
\text { fetales }\end{array}$ & Cesárea & $\begin{array}{l}\text { Hematoma de vena } \\
\text { umbilical } \\
\text { Arterias umbilicales } \\
\text { comprimidas, pero } \\
\text { permeables }\end{array}$ & $\begin{array}{l}\text { Favorable } \\
\text { (Apgar 7/9) }\end{array}$ & 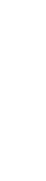 \\
\hline & 22 & 40 & $\begin{array}{l}\text { Disminución de } \\
\text { movimientos } \\
\text { fetales }\end{array}$ & Cesárea & $\begin{array}{l}\text { Vena y arterias } \\
\text { umbilicales comprimidas, } \\
\text { pero permeables }\end{array}$ & $\begin{array}{l}\text { Favorable } \\
\text { (Apgar 2/6/8) }\end{array}$ & (2) \\
\hline & 39 & 38 & $\begin{array}{l}\text { Disminución de } \\
\text { movimientos } \\
\text { fetales }\end{array}$ & Cesárea & $\begin{array}{l}\text { Vena y arterias } \\
\text { umbilicales permeables }\end{array}$ & $\begin{array}{l}\text { Favorable } \\
\text { (Apgar 8/9) }\end{array}$ & 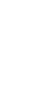 \\
\hline $\begin{array}{l}\text { Barbati, et al. }{ }^{14} \\
(2009)\end{array}$ & 44 & 40 & $\begin{array}{l}\text { Disminución de } \\
\text { movimientos } \\
\text { fetales } \\
\text { Baja } \\
\text { variabilidad en } \\
\text { RCTG }\end{array}$ & Cesárea & $\begin{array}{l}\text { Extravasación de sangre } \\
\text { en la gelatina de Wharton } \\
\text { circundante causada por } \\
\text { la rotura de una arteria } \\
\text { umbilical dilatada }\end{array}$ & $\begin{array}{l}\text { Favorable } \\
\text { (Apgar 6/9) }\end{array}$ & 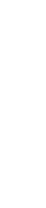 \\
\hline $\begin{array}{l}\text { Kumar, et al. }{ }^{12} \\
\text { (2012) }\end{array}$ & 31 & 36 & $\begin{array}{l}\text { Disminución de } \\
\text { movimientos } \\
\text { fetales }\end{array}$ & Cesárea & $\begin{array}{l}\text { Una de las arterias } \\
\text { umbilicales rotas con } \\
\text { inflamación aguda leve y } \\
\text { células musculares } \\
\text { necróticas en su pared }\end{array}$ & $\begin{array}{l}\text { Taquicardia, } \\
\text { taquipnea e } \\
\text { insuficiência } \\
\text { renal aguda } \\
\text { por necrosis } \\
\text { tubular aguda }\end{array}$ & 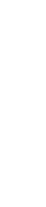 \\
\hline $\begin{array}{l}\text { Jouanelle, } \\
\text { et al. }{ }^{13} \text { (2012) }\end{array}$ & Desconocida & A término & $\begin{array}{l}\text { Disminución de } \\
\text { movimientos } \\
\text { fetales } \\
\text { Deceleraciones } \\
\text { en RCTG }\end{array}$ & Cesárea & Desconocido & $\begin{array}{l}\text { Muerte fetal al } \\
\text { sexto día de } \\
\text { vida }\end{array}$ & 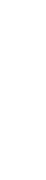 \\
\hline $\begin{array}{l}\text { Tonni, et al. }{ }^{10} \\
\text { (2015) }\end{array}$ & 19 & A término & $\begin{array}{l}\text { Bradicardia } \\
\text { fetal en RCTG }\end{array}$ & Parto vaginal & $\begin{array}{l}\text { Rotura de pared de la } \\
\text { vena umbilical } \\
\text { Una arteria umbilical con } \\
\text { disección y rotura de las } \\
\text { fibras elásticas } \\
\text { Corioamnionitis difusa } \\
\text { (infección por Escherichia } \\
\text { coli) }\end{array}$ & $\begin{array}{l}\text { A los } 4 \text { años: } \\
\text { tetraplejía, } \\
\text { convulsiones, } \\
\text { sordera y } \\
\text { ceguera }\end{array}$ & 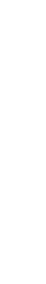 \\
\hline $\begin{array}{l}\text { Abraham, et al. }{ }^{11} \\
(2015)\end{array}$ & 27 & 35 & $\begin{array}{l}\text { Muerte fetal } \\
\text { intraútero }\end{array}$ & Parto vaginal & Corioamnionitis difusa & & $\begin{array}{l}\bar{c} \\
0 \\
0\end{array}$ \\
\hline $\begin{array}{l}\text { McAdams y } \\
\text { Chabra8 (2016) }\end{array}$ & 32 & A término & Sin hallazgos & Parto vaginal & Desconocido & Favorable & "ะ ఝ \\
\hline $\begin{array}{l}\text { Hooper y } \\
\text { Sebire15 (2016) }\end{array}$ & Desconocida & A término & Sin hallazgos & Parto vaginal & Desconocido & Favorable & $\frac{0}{\circ}$ \\
\hline $\begin{array}{l}\text { Arora, et al. }{ }^{16} \\
(2017)\end{array}$ & Desconocida & 39 & Sin hallazgos & Parto vaginal & Desconocido & $\begin{array}{l}\text { Favorable } \\
\text { (Apgar 8/9) }\end{array}$ & $\frac{0}{2}$ \\
\hline $\begin{array}{l}\text { Scutiero, et al. }{ }^{17} \\
(2018)\end{array}$ & 29 & 41 & Sin hallazgos & Parto vaginal & $\begin{array}{l}\text { Edema y hemorragia } \\
\text { extensa de la gelatina de } \\
\text { Wharton } \\
\text { Luz de la vena ocluida } \\
\text { por material hemático } \\
\text { coagulado }\end{array}$ & $\begin{array}{l}\text { Muerte } \\
\text { perinatal }\end{array}$ & 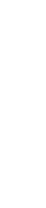 \\
\hline $\begin{array}{l}\text { Skowronek, } \\
\text { et al. }{ }^{18}(2018)\end{array}$ & Desconocida & 41 & $\begin{array}{l}\text { Bradicardia } \\
\text { fetal en RCTG }\end{array}$ & Cesárea & $\begin{array}{l}\text { No alteraciones } \\
\text { histopatológicas }\end{array}$ & $\begin{array}{l}\text { Muerte } \\
\text { perinatal }\end{array}$ & 엄 \\
\hline $\begin{array}{l}\text { Mota, et al. }{ }^{19} \\
(2019)\end{array}$ & Desconocida & 39 & Sin hallazgos & Parto vaginal & $\begin{array}{l}\text { No alteraciones } \\
\text { histopatológicas }\end{array}$ & Favorable & ○ิ \\
\hline $\begin{array}{l}\text { Pezron, et al. }{ }^{20} \\
(2020)\end{array}$ & Desconocida & A término & Sin hallazgos & Desconocido & Desconocido & Favorable & \\
\hline
\end{tabular}

RCTG: registro cardiotocográfico. 
hidrorrea. Se complementó la exploración con una ecografía transabdominal, en la cual se vio un feto en presentación cefálica, con peso fetal estimado de $4500 \mathrm{~g}$, con movimientos de tronco y extremidades positivos, placenta normoinserta en la cara posterior, sin signos de desprendimiento y una cantidad de líquido amniótico normal. Se procedió a la monitorización fetal continua, en la que se objetivó una frecuencia cardiaca fetal media de 160 latidos por minuto (I.p.m.), sin aceleraciones ni deceleraciones, con una variabilidad en el límite inferior de la normalidad (Fig. 1). Se decidió entonces llevar a cabo un estudio Doppler, que mostró como único hallazgo relevante un conducto venoso por encima del percentil 95. Tras consensuar la actitud obstétrica, y atendiendo a las recomendaciones actuales de la Sociedad Española de Obstetricia y Ginecología respecto a la vía de elección del parto en las gestantes diabéticas con un peso fetal estimado por encima de $4500 \mathrm{~g}$, se decidió realizar una cesárea de urgencia, en la que nació un varón de $4560 \mathrm{~g}$, con un test de Apgar 8/10/10 y un pH de arteria umbilical de 7.08.

Tras el alumbramiento se identificó un hematoma de aproximadamente $6 \mathrm{~cm}$ en la zona central del cordón umbilical, cercano a la inserción de este (Fig. 2). El estudio histológico describió una placenta de $650 \mathrm{~g}$, acorde con una edad gestacional al término, sin otras alteraciones destacables. El cordón presentó dos falsos nudos en su porción más distal, con tres luces vasculares, y una gran colección hemática en la cara fetal que ocupaba un $10 \%$ del volumen total placentario.

Fue ingresado en la unidad de neonatología por el hallazgo de un soplo cardiaco. Tras su estudio, se diagnosticó miocardiopatía hipertrofia obstructiva, actualmente en seguimiento periódico, sin otras alteraciones.

\section{Discusión}

El hematoma del cordón umbilical, aunque es muy poco frecuente, es una complicación realmente grave del embarazo. En 1940, Dippers, et al., con 36 casos, describieron la serie más grande publicada hasta ahora. Se calculó una incidencia de 1 en 5505 nacimientos y la tasa general de muerte perinatal fue de aproximadamente el $50 \%$.

La etiología exacta del hematoma de cordón umbilical aún se desconoce. Se han propuesto muchas teorías, pero sin llegar a un consenso final. Probablemente sea una combinación de diferentes factores lo que conduzca a su aparición. La posibilidad de una anomalía cromosómica es remota. Berg, et al. ${ }^{6}$ reportaron un caso de aneurisma del cordón umbilical en un feto

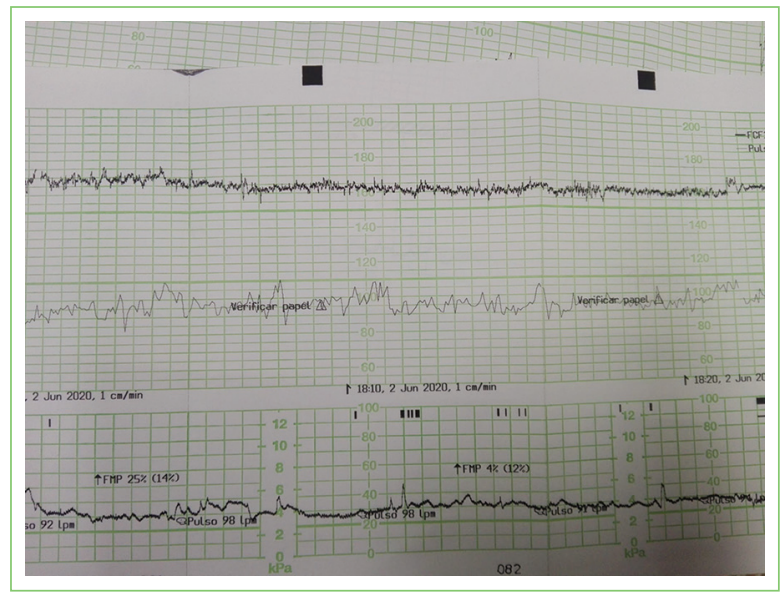

Figura 1. Monitorización fetal. Frecuencia cardiaca fetal media en torno a 160 latidos por minuto (I.p.m.), sin aceleraciones ni deceleraciones, con una variabilidad de 5 I.p.m., es decir, en el límite inferior de la normalidad.

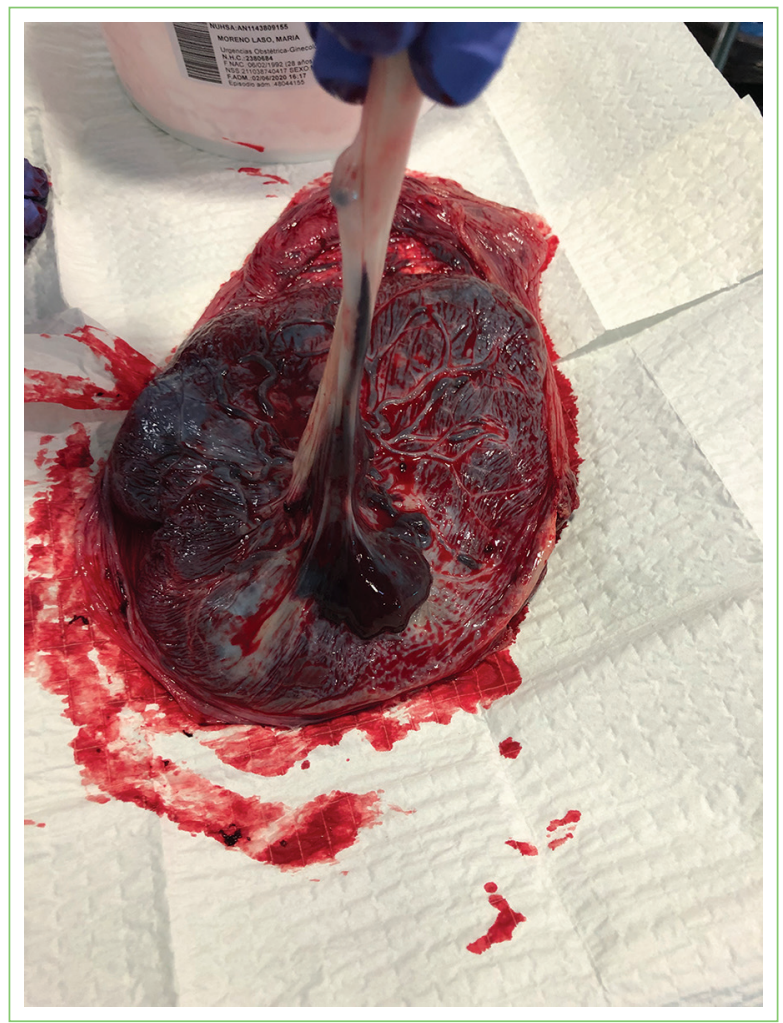

Figura 2. Hematoma de cordón umbilical macroscópicamente visible tras el alumbramiento.

con trisomía 18. Como nuestro feto tenía una apariencia normal, no consideramos necesario llevar a cabo ningún estudio cromosómico. 
Los factores de riesgo para el hematoma espontáneo del cordón umbilical son varios. Se incluyen anomalías morfológicas del cordón umbilical (tanto en longitud como en grosor), nudos verdaderos, prolapso, tracción o torsión del cordón, inserción velamentosa del cordón, anomalías de la pared de los vasos, quistes del cordón umbilical, traumatismo abdominal durante la gestación, infecciones (corioamnionitis y funisitis) y déficit de gelatina de Wharton. También se han informado causas iatrogénicas secundarias a amniocentesis, transfusiones intraútero y cordocentesis diagnóstica?.

McAdams y Chabra $^{8}$ informaron que la macrosomía también podría considerarse relacionada con la aparición del hematoma de cordón umbilical. ClermontHama, et al. ${ }^{9}$ describieron que el oligohidramnios del tercer trimestre (30.8 vs. $2.6 \%$; odds ratio [OR]: 16.9 ; $\mathrm{p}=0.01$ ), la amniocentesis del segundo trimestre (33.3 vs. $5.1 \%$; OR: 9.3; $p=0.02$ ) y la reducción de los movimientos fetales percibidos por la madre (35.7 vs. $7.7 \% ; p=0.02)$ se asociaban significativamente con hematomas espontáneos del cordón umbilical. En nuestro caso, la longitud del cordón estaba dentro del rango normal y no se observaron antecedentes de amniocentesis ni de traumatismo abdominal durante el embarazo. El recién nacido era un feto macrosómico, $y$ es el segundo caso descrito que apoya esta asociación.

El sangrado espontáneo en el cordón umbilical se debe a una interrupción de la pared del vaso a través de la cual, en la mayoría de los casos, se produce una extravasación de sangre en la gelatina de Wharton. Una presión intravascular alta podría estar implicada en su formación. El hematoma puede comprometer la circulación materno-fetal al comprimir los vasos (arterias y venas umbilicales), con hipoxia fetal posterior, o por la pérdida de sangre dentro del propio cordón con anemia, lo que conduce a asfixia perinatal y muerte fetal ${ }^{8}$. En nuestro análisis de 15 casos, dos mostraron evidencia de corioamnionitis ${ }^{10,11}$, uno tuvo arteria umbilical única e inserción del cordón marginal con avulsión espontánea de la arteria umbilical ${ }^{12}$, otro se asoció a déficit congénito de factor $\mathrm{VII}^{13}$ y en 11 casos no se informó ninguna condición patológica $^{5,8,14-20}$. La rotura de la vena es más común que la de las arterias $(1: 10)^{13}$. Los hematomas del cordón umbilical pueden localizarse en cualquier punto de su longitud, pero la ubicación más común es el extremo fetal ${ }^{18}$. En nuestro caso, el hematoma se encontraba en el extremo placentario, hallazgo que rara vez se informa en la literatura.
El diagnóstico suele realizarse tras el nacimiento, pero en algunos casos puede hacerse prenatalmente mediante ecografía Doppler, evaluando el cordón y el flujo sanguíneo en los vasos umbilicales ${ }^{16,17}$. Los hematomas del cordón umbilical pueden surgir durante el embarazo, lo que puede conducir a la muerte fetal ${ }^{11}$, o pueden ocurrir con mayor frecuencia durante el trabajo de parto, lo que genera sufrimiento fetal y requiere una actuación obstétrica inmediata. De los 15 casos reportados en los 13 estudios de esta revisión, cuatro se presentaron como muertes perinatales, tres como muerte fetal y uno en el día 6 de vida ${ }^{11,13,17,18}$. De los 11 nacidos vivos, nueve fueron al término de la edad gestacional y dos fueron prematuros 5,8,10,12,14-16,19,20. Además, ocho casos presentaron como motivo de consulta una disminución de la percepción de los movimientos fetales ${ }^{5,11-14,17}$. El registro cardiotocográfico detectó anormalidades de la frecuencia cardiaca fetal en siete casos $^{5,12-14,18}$. En cinco casos no se produjeron cambios en los movimientos fetales ni anormalidades en la frecuencia cardiaca fetal. En el caso que nos ocupa, la paciente consultó en urgencias por disminución de la percepción de movimientos fetales de 6 horas de evolución. El registro cardiotocográfico mostró una frecuencia cardiaca fetal de 160 I.p.m., con una variabilidad disminuida, sin ascensos ni deceleraciones.

El examen detallado de la placenta y del cordón umbilical confirmó la presencia de un hematoma en los 15 casos descritos. Durante el examen macroscópico, el cordón umbilical puede tener un aspecto anormal, con decoloración rojo oscuro y un grosor notablemente aumentado ${ }^{15}$. También puede tener un bulto oscuro y una decoloración azulada ${ }^{8}$. En otros casos, la inspección del funículo no muestra ningún hallazgo patológico y el hematoma solo es evidente cuando se corta. Por lo tanto, en general, se puede decir que el diagnóstico de hematoma de cordón puede estar infraestimado porque, como regla general, la sección múltiple del funículo no siempre se lleva a cabo.

El examen histopatológico de la placenta y del cordón objetivó la presencia de un hematoma y mostró evidencia de corioamnionitis en dos $\operatorname{casos}^{10,11}$. El examen histológico de la porción del cordón afectada por el hematoma puede mostrar infiltración hemorrágica perivascular, vasos umbilicales comprimidos por el derrame hemorrágico, fisuras de la pared venosa, alteraciones de la íntima y de la túnica media con la pared de los vasos marcadamente adelgazada por la reducción de la componente muscular, y también infiltración leucocitaria inflamatoria moderada de las paredes vasculares umbilicales ${ }^{17}$. 
En cuanto al manejo terapéutico después del parto, los hematomas del cordón umbilical generalmente no necesitan atención especializada y pueden dejarse caer con el cordón umbilical ${ }^{16}$.

\section{Conclusiones}

El hematoma del cordón umbilical, aunque es muy poco frecuente, es una complicación realmente grave del embarazo. Su etiología exacta aún no se conoce. El diagnóstico se realiza habitualmente de forma posnatal, dada la dificultad para su confirmación ecográfica. El pronóstico puede ser de morbimortalidad fetal importante por la posible hipoxia fetal provocada, secundaria a la compresión de los vasos umbilicales. Así pues, resulta imprescindible informar todos los casos nuevos de hematoma del cordón umbilical para mejorar el conocimiento de los obstetras acerca de los factores predisponentes, el diagnóstico prenatal y el manejo clínico, de modo que se puedan tomar las medidas oportunas para evitar el sufrimiento fetal o la muerte fetal intrauterina, y mejorar el diagnóstico prenatal.

\section{Financiamiento}

No se ha obtenido ninguna fuente de financiamiento.

\section{Conflicto de intereses}

No se ha declarado ningún conflicto de intereses.

\section{Responsabilidades éticas}

Protección de personas y animales. Los autores declaran que para esta investigación no se han realizado experimentos en seres humanos ni en animales.

Confidencialidad de los datos. Los autores declaran que han seguido los protocolos de su centro de trabajo sobre la publicación de datos de pacientes.

Derecho a la privacidad y consentimiento informado. Los autores han obtenido el consentimiento informado de los pacientes y/o sujetos referidos en el artículo. Este documento obra en poder del autor de correspondencia.

\section{Bibliografía}

1. Yoshida K, Furuhashi M, Nakagawa A, Kidokoro K, Kuno N, Ishikawa K. Umbilical cord hematoma: association with corioamnionitis and funisitis. Acta Obstet Gynecol Scand. 2007;86:895-6.

2. Sepúlveda W, Wong AE, González R, Vásquez P, Gutiérrez J. Fetal death due to umbilical cord hematoma: a rare complication of umbilical cord cyst. J Matern Fetal Neonatal Med. 2005;18:387-90.

3. González Santacruz M, Blanco Alemany E, García Martínez MR. Hematoma espontáneo de cordón umbilical. An Pediatr (Barc). 2008;69:482-3.

4. Gualandri G, Rivasi F, Santunione AL, Silingardi E. Spontaneous umbilical cord hematoma: an unusual cause of fetal mortality: a report of 3 cases and review of the literature. Am J Forensic Med Pathol. 2008;29:185-90.

5. Towers CV, Juratsch CE, Garite TJ. The fetal heart monitor tracing in pregnancies complicated by a spontaneous umbilical cord hematoma. J Perinatol. 2009;29:517-20.

6. Berg C, Geipel A, Germer U, Gloeckner-Hofmann K, Gembruch U. Prenatal diagnosis of umbilical cord aneurysm in a fetus with trisomy 18. Ultrasound Obstet Gynecol. 2001;17:79-81.

7. Nappi L, Trezza F, Bufo P, Riezzo I, Turillazzi E, Borghi C, et al. Classification of stillbirths is an ongoing dilemma. J Perinat Med. 2016;44:837-43.

8. McAdams RM, Chabra S. Umbilical cord haematoma and adrenal haemorrhage in a macrosomic neonate with anaemia. BMJ Case Rep. 2016;2016:bcr2015214140.

9. Clermont-Hama Y, Thibouw K, Devisme L, Franquet-Ansart H, Stichelbout M, Subtil D. Risk factors for spontaneous hematoma of the umbilical cord: a case-control study. Placenta. 2020;99:152-6.

10. Tonni G, Bonasoni MP, De Felice C, Rossi A, Tonni S. Histopathological findings in spontaneous hematoma of the umbilical cord: severe hypoxic-ischemic encephalopathy in a term survived newborn. Am J Forensic Med Pathol. 2015;36:254-6.

11. Abraham A, Rathore S, Gupta M, Benjamin SJ. Umbilical cord haematoma causing stillbirth — a case report. J Clin Diagn Res. 2015;9:QD01-2.

12. Kumar A, Kaplan $C$, Mokrian S, Ogburn P. Intact newborn survival after spontaneous umbilical cord vascular rupture before labor. Obstet Gynecol. 2012;120(2 Pt 2):489-90.

13. Jouannelle $C$, Giansily-Blaizot M, Monpoux F, Casagrande F, Poirée M, Bérard E. Spontaneous umbilical cord haematoma and congenital factor VII deficiency. Haemophilia. 2012;18:e24-5.

14. Barbati A, Cacace MG, Fratini D, Ceccarelli T, Capanna F, Di Renzo GC. Umbilical cord haematoma with altered fetal heart rate. J Obstet Gynaecol. 2009;29:150-1.

15. Hooper KE, Sebire P. Spontaneous umbilical cord haematoma. Arch Dis Child Fetal Neonatal Ed. 2016;101:F332.

16. Arora PK, Mohandas S, McAndrew S, Karody V. Spontaneous umbilical cord hematoma. J Pediatr. 2017;184:233-233.e1.

17. Scutiero G, Bernardi G, lannone P, Nappi L, Morano D, Greco P. Umbilical cord hematoma: a case report and review of the literature. Obstet Gynecol Int. 2018;2018:2610980.

18. Skowronek R, Zamłyński M, Kajor M, Zamłyński J, Chowaniec M. Spontaneous umbilical cord hematoma as a clinical and forensic medical problem in case of suspected obstetrician's medical malpractice. Ginekol Pol. 2018;89:642-3.

19. Mota F, Oliveira N, Fonseca M, Mimoso G. Spontaneous umbilical cord hematoma. BMJ Case Rep. 2019;12:e229952.

20. Pezron J, Tellai L, Tourneux P. Spontaneous umbilical cord hematoma with a favorable outcome. Arch Pediatr. 2020;27:380-2. 